\title{
Investigating inherited influences on anthropometric and metabolic parameters in adult twins
}

\author{
K. Bermingham, R. Barron, L. Brennan, E. Gibney, M. Gibney, M. Ryan and A. O’Sullivan \\ Institute of Food and Health, University College Dublin, Republic of Ireland
}

Twin research is based on the foundation that all human traits are heritable and differentially influenced by our genes. Twin studies have demonstrated that genetic factors contribute to variations in anthropometric and metabolic parameters ${ }^{(1)}$. For example, high heritability estimates for height have been reported and clearly demonstrated by increased intra-pair similarity in twins ${ }^{(2)}$. In this study we aimed to investigate inherited influences on a range of anthropometric and metabolic markers in a population of healthy adult twins.

A cohort of 44 monozygotic (MZ) and 21 dizygotic $(\mathrm{DZ})$ twin pairs (total $n=130$ ) aged 18-65 years were recruited into the UCD twin study. Data were collected on body composition and from fasting blood samples including total cholesterol, HDL-cholesterol, LDL-cholesterol, TAGS, NEFAs, glucose and insulin. For 15 measured traits, partial correlations were calculated to test for inherited influences by comparing whether intra-pair similarity was greater in MZ twins than DZ twins. General linear models (GLM) were used to assess differences between $\mathrm{MZ}$ and DZ correlations controlling for age, gender and interactions. Phenotypic profile similarity across twins was examined by combining correlations of all 15 traits. Ethics was obtained from UCD Human Research Ethics Committee.

Strong intra-pair similarities were observed with all traits. Evidence of genetic effects on height, body mass, BMI, waist, body fat \% and glucose was indicated, as the MZ correlations were significantly greater than the DZ correlations (Table 1). The average similarity across the full phenotypic profile was higher for MZ twin pairs than for DZ twin pairs (Fig 1).

Table 1. Partial correlations, age and gender controlled.

\begin{tabular}{|c|c|c|c|}
\hline Variable & MZ twins & DZ twins & $\mathrm{P}$-value \\
\hline Height (m) & 0.947 & $0 \cdot 371$ & $0.041 *$ \\
\hline Body mass $(\mathrm{kg})$ & 0.836 & $0 \cdot 294$ & $0 \cdot 010^{*}$ \\
\hline BMI $(\mathrm{kg} / \mathrm{m} 2)$ & $0 \cdot 850$ & $0 \cdot 092$ & $0 \cdot 000^{*}$ \\
\hline Waist $(\mathrm{cm})$ & 0.759 & 0.405 & $0.003^{*}$ \\
\hline Hip (cm) & $0 \cdot 848$ & 0.431 & 0.079 \\
\hline WHR $(\mathrm{cm} / \mathrm{cm})$ & $0 \cdot 690$ & 0.077 & 0.066 \\
\hline Body fat $(\%)$ & 0.782 & $0 \cdot 280$ & $0 \cdot 004 *$ \\
\hline $\mathrm{SBP}(\mathrm{mmHg})$ & 0.691 & $0 \cdot 395$ & 0.135 \\
\hline Cholesterol $(\mathrm{mmol} / \mathrm{l})$ & $0 \cdot 773$ & 0.635 & 0.434 \\
\hline HDL (mmol/l) & $0 \cdot 513$ & 0.515 & 0.663 \\
\hline $\operatorname{LDL}(\mathrm{mmol} / \mathrm{l})$ & 0.824 & 0.577 & $0 \cdot 196$ \\
\hline TAGS (mmol/l) & $0 \cdot 549$ & $0 \cdot 353$ & $0 \cdot 349$ \\
\hline NEFA $(\mathrm{mmol} / \mathrm{l})$ & 0.441 & $-0 \cdot 094$ & $0 \cdot 160$ \\
\hline Glucose $(\mathrm{mmol} / \mathrm{l})$ & $0 \cdot 556$ & $-0 \cdot 020$ & $0 \cdot 004 *$ \\
\hline Insulin (mU/l) & $0 \cdot 327$ & 0.048 & 0.396 \\
\hline
\end{tabular}

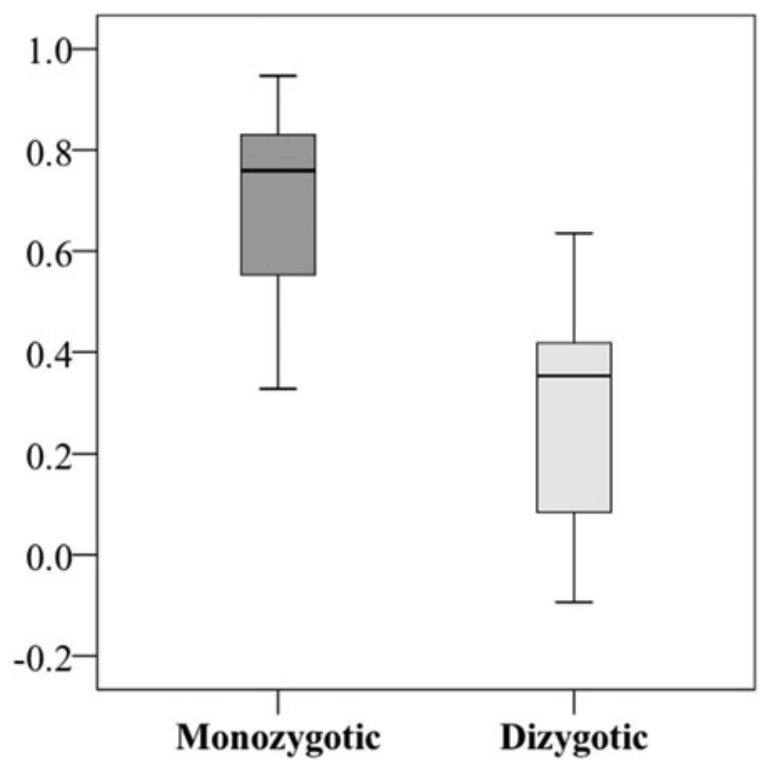

Fig. 1. Phenotypic profile correlations

In conclusion, phenotypic traits and the phenotypic profile are more similar for MZ twins demonstrating the importance of genetic factors. More advanced twin modeling will examine the contribution of genetic and environmental influences on anthropometric and metabolic variance. This knowledge can be coupled with more advanced technologies in the future to interpret complex phenotypes such as the metabolome.

1. Polderman TJ, Benyamin B, De Leeuw CA, et al. (2015) Nat Genet. 47, 702-709.

2. Silventoinen K. et al. (2003) Twin Res. 6, 399-408. 